10

\title{
Key Factors for Tuning Au Self-Assembling SERS Films: from Properties to Structure*
}

\author{
(C) K.A. Khnykina, M.A. Baranov, A.A. Babaev, A.V. Baranov, and K.V. Bogdanov \\ Center of Information Optical Technologies, ITMO University, \\ 197101 St. Petersburg, Russia \\ Te-mail: kirw.bog@gmail.com
}

Received November 18, 2020

Revised November 18, 2020

Accepted December 4, 2020

We studied self-assembled plasmonic films (SPF) based on gold nanoparticles (Au-NPs). We recorded the intensity distribution maps (IDM) with an area of $2025 \mu \mathrm{m}^{2}(9 \times 9$ points). We have observed uniform enhancement across the entire surface of SPF. Therefore, we used the parameters average intensity of IDM and IDM gate when we optimized the synthesis of SPF. Average intensity is the average intensity value over 81 spectra of the IDM. The gate is the span excluding $10 \%$ of the outliers. We have discovered that the maximum average intensity was observed at a concentration of $1.00 \cdot 10^{-5} \mathrm{M}$. A decrease in the concentration of Au-NPs leads to a decrease in the average intensity. A simultaneous decrease in TOABr and $\mathrm{Au}-\mathrm{NPs}$ concentration also leads to a decrease in the average intensity. The film obtained by the optimal synthesis method was characterized using SEM and AFM.

Keywords: SERS, Au-NPs, self-assembling.

* Полный текст статьи опубликован в „Optics and Spectroscopy“ 2021 V. 129. N 4. 